Research Paper

\title{
Epidemiology of cancer in end-stage renal disease dialysis patients: a national cohort study in Taiwan
}

\author{
Chih-Chiang Chien'1,2, Ming-Ming Han³, Yu-Hsien Chiu', Jhi-Joung Wang5, Chin-Chen Chu',7, Chien-Ya \\ Hung ${ }^{2}$, Yih-Min Sun ${ }^{8}$, Nai-Cheng Yeh ${ }^{9}$, Chung-Han Ho ${ }^{5}$, Chih-Ching Lin ${ }^{10}$, Hao-Yun Kao ${ }^{\star 凶}$, Shih-Feng \\ Weng ${ }^{4}$ \\ 1. Department of Nephrology, Chi-Mei Medical Center, Tainan, Taiwan. \\ 2. Department of Food Nutrition, Chung-Hwa University of Medical Technology, Tainan, Taiwan. \\ 3. Department of Anesthesiology, Anhui Provincial Hospital, Anhui Medical University, Hefei, China. \\ 4. Department of Healthcare Administration and Medical Informatics, Kaohsiung Medical University, Kaohsiung, Taiwan. \\ 5. Department of Medical Research, Chi-Mei Medical Center, Tainan, Taiwan. \\ 6. Department of Recreation and Health-Care Management, Chia Nan University of Pharmacy and Science, Tainan, Taiwan. \\ 7. Department of Anesthesiology, Chi-Mei Medical Center, Tainan, Taiwan. \\ 8. Department of Occupational Safety and Health, Chung Hwa University of Medical Technology, Tainan, Taiwan. \\ 9. Department of Endocrinology and Metabolism, Chi-Mei Medical Center, Tainan, Taiwan. \\ 10. Department of Nephrology, Taipei Veterans General Hospital, Taiwan. \\ * These authors equally contributed to this work. \\ $\triangle$ Corresponding authors: Department of Healthcare Administration and Medical Informatics, Kaohsiung Medical University, Kaohsiung, Taiwan. Email: \\ sfweng@kmu.edu.tw (SF. Weng), haoyun@kmu.edu.tw (HY. Kao).
}

(c) Ivyspring International Publisher. This is an open access article distributed under the terms of the Creative Commons Attribution (CC BY-NC) license (https://creativecommons.org/licenses/by-nc/4.0/). See http://ivyspring.com/terms for full terms and conditions.

Received: 2016.06.20; Accepted: 2016.10.29; Published: 2017.01.01

\begin{abstract}
The incidence and mortality of site-specific cancers in patients with end-stage renal disease (ESRD) on maintenance dialysis have been rarely studied for Asian populations. We tapped Taiwan's National Health Insurance Research Database to identify and recruit patients starting maintenance dialysis between 1999 and 2004. They were followed from initiation of dialysis until death, discontinuation of dialysis, or the end of 2008. We calculated the survival rate and mortality risk of dialysis patients with cancer. Of 40,833 dialysis patients, 2352 (5.8\%) had been newly diagnosed with cancer. Being older, being male, and having chronic liver disease were factors associated with a higher risk for new cancer in ESRD dialysis patients. In men, liver cancer $(20.63 \%)$ was the most frequent, followed by cancers of the bladder (16.88\%) and kidney (11.61\%). In women, bladder cancer (25.57\%) was the most frequent, followed by cancers of the kidney (16.31\%) and breast (11.20\%). The 5-year survival rates for kidney and bladder cancer were higher than for other cancers; the survival rates for lung, stomach, and liver cancer were lower. In conclusion, the distribution of site-specific cancer was different between men and women in patients with ESRD on dialysis. More attention should be paid to teaching dialysis patients how to avoid the well-known cancer risks and carcinogens and individualized regular cancer screenings.
\end{abstract}

Key words: Cancer, end-stage renal disease, incidence, dialysis.

\section{Introduction}

Cancer is a leading cause of mortality worldwide. In 2008, it accounted for about $13 \%$ of all deaths [1]. Patients with chronic kidney disease (CKD), both those who were on long-term dialysis [2] and those who were not [3-6], have a higher-than-normal cancer risk.

Studies [4-6] have reported significant differences in the incidence rate of various cancers between ESRD patients and the general population.
Kidney, urinary tract, thyroid, tongue, liver, and cervical cancers are clearly more common among ESRD patients [3-5]. However, there are some conflicting data with regard to gender discrepancy. A large international collaborative study [3] reported that the overall risk for cancer was higher in women than in men during dialysis, but other investigations $[6,7]$ have reported contradictory findings. Thus far, very few studies have examined the survival rate of 
ESRD patients on long-term renal dialysis for those with various types of cancer [6]. Furthermore, little is known about predictors of mortality in these patients, because acquiring such data requires studies of large populations and long follow-up periods.

The incidence and prevalence rates of ESRD patients on dialysis are high in Taiwan [8]. In addition, the Asia-Pacific area, including Taiwan, is highly endemic for chronic viral hepatitis [9, 10]. Although surveys on cancer in ESRD dialysis patients have been published in various Western countries, published information on this topic for Asian populations is sparse [6]. Un this study, we used a large data set from Taiwan's National Health Insurance Research Database (NHIRD) to perform a long-term population-based cohort study to identify risk factors, and to estimate the all-cause mortality rate in ESRD dialysis patients with cancer.

\section{Materials and Methods}

\section{Database}

The Taiwan National Health Insurance (NHI) program, which provides compulsory universal health insurance for almost all Taiwan`s residents, was implemented in 1995 . With the exception of prison inmates, all residents in Taiwan are enrolled in the program. All contracted medical institutions must submit standard claims documents for medical expenses on a computerized form. All chronic dialysis patients are covered by the NHI program; so, NHIRD include the data of all dialysis patients in Taiwan. Data for this cohort study were obtained from the NHIRD, which was released for research purposes by the Taiwan`s National Health Research Institute. The NHIRD, which includes nearly all (99\%) inpatient and outpatient medical benefit claims for the Taiwan's population of 23 million people, is one of the largest and most comprehensive databases in the world and has been used extensively in various studies [11-16]. The NHIRD, in which patient identification numbers are encrypted, contains patients claims data including gender, birthday, dates of admission and discharge, medical institutions providing the services, the ICD-9-CM (International Classification of Diseases, 9th Revision, Clinical Modification) diagnostic (up to five) and procedure codes (up to five), and outcome. We used all claims reported from 1998 to 2008 including those of all ESRD dialysis patients. All datasets were interlinked through each subject's unique personal identification number.

\section{Patient selection and definition}

Using the NHIRD, we enrolled ESRD patients who started maintenance dialysis between January 1 , 1999, and December 31, $2004(n=43,361)$. ESRD patients were defined as being on maintenance dialysis if they had received dialysis for more than 90 days. Excluded were patients $(n=2528)$ on dialysis after a transplantation $(n=2223)$ and patients for whom the diagnosis of cancer preceded dialysis ( $\mathrm{n}=$ 273). Patients were followed from the first reported date of dialysis to the date of renal transplantation, death, end of dialysis, or December 31, 2008. A total of 40,833 incident dialysis patients were analyzed in the study. The Institutional Review Board of Chi Mei Medical Center approved this study and waived the requirement of informed consent because all personal information in the NHIRD was de-identified, which protects the privacy of the patients and their families. The protocol of this study was carried out in accordance with the approved guidelines.

In Taiwan, the NHI Bureau issues major illness/injury certificates to all patients who had a malignant neoplasm. Patients with these certificates are exempt from copayments to the NHI if they are admitted for an illness associated with the related malignancy. We identified patients with malignancies (ICD-9: 140-208) and confirmed that they had used their major illness/injury certificates. The definition of a newly diagnosed malignant neoplasm was a malignancy diagnosed more than 90 days after dialysis had begun. A total of 2352 patients had newly diagnosed malignant neoplasms.

Medical records from the NHIRD were used to obtain each patient's gender, age, date of birth, starting and ending dates of dialysis, cancer diagnosis date, type of cancer, time interval from starting dialysis to the development of cancer, time interval from the development of cancer to death, and baseline comorbidities. The ICD-9-CM codes used to define each comorbidity were as follows: (1) diabetes mellitus (DM) (250.x x, 357.2, 362.0x, 366.41); (2) hypertension (HTN) (362.11, 401.x -405.x, 437.2); (3) Cardiovascular diseases (CVD) (410.xx - 414.xx); (4) Chronic lung disease (490 - 496.xx, 500-505.xx, 506.4.xx); (5) Chronic liver disease (570, 571, 572.1, 572.4, 573.1-573.3); and (6) Congenital renal and urinary tract disease (753.0, $\left.753.1^{*}, 753.2^{*}, 753.3\right)$. Those comorbidities were identified according to one of the definitions below: (1) Diagnostic codes in outpatient visits if the patient had an initial diagnosis at any time the year leading up to beginning of dialysis and then experienced two or more additional diagnoses within the subsequent twelve months; (2) Diagnostic codes in hospitalization databases at least one time within the year leading up to start of dialysis.

\section{Statistical Analysis}

All values are expressed as means \pm standard deviation (SD) or incidence (\%). Comparisons of 
groups with or without a diagnosis of new cancer were done using $\chi^{2}$ tests for categorical variables. We calculated the number of person-years as the risk from the date of the first dialysis until the date of the diagnosis of new cancer, renal transplantation, death, end of dialysis, or December 31, 2008. The proportion of patients free from new cancer was calculated using the Kaplan-Meier method. Hazard ratios (HRs) and 95\% confidence intervals (CIs) were calculated for each factor using a Cox univariate analysis. Covariates, including established risk factors, were also assessed using Cox multivariable regression analysis to determine significant predictors for new cancers.

In the group with new cancer, the cumulative survival rate after the new cancer had been diagnosed was also calculated using the Kaplan-Meier method. HRs and 95\% CIs were used to determine significant predictors for mortality. All statistical analyses were done with the Statistical Package for Social Sciences for Windows version 17.0 (SPSS Inc; Chicago, IL, USA).

\section{Results}

\section{Baseline demographic and risk factors for new Cancer}

Of 40,833 dialysis patients, 2352 (5.8\%) had newly diagnosed cancer (Table 1). The 1-, 5-, and 9 -year cumulative cancer rates were $0.1 \%, 0.2 \%$ and $0.5 \%$ in males, and $0.1 \%, 0.2 \%$, and $0.5 \%$ in females (log-rank test: $\mathrm{P}<0.113$ ) (Figure 1). The mean duration from the initiation of dialysis to new cancer was 41.29 \pm 26.58 months; the median was 37.37 months (25th-75th percentile, 19.37 - 58.19). Being older, being male, and having chronic liver disease were independent risk factors for new cancer in ESRD dialysis patients (Table 1). Compared to patients 18-35 years, those 35-64 years old were three times more likely to develop cancer (HR 3.42 [95\% CI 2.60 - 4.48]) and those $\geq 65$ years old had nearly a fivefold increase in cancer (HR 4.73 [95\% CI 3.59 - 6.25]). Patients with chronic liver disease had a $76 \%$ higher risk for new cancer (HR: 1.756, 95\% CI: 1.550-1.990); while, ESRD patients with DM, HTN, or CVD had lower risk of new cancer after beginning dialysis.

\section{Site-specific cancer analyses}

The mean interval from beginning dialysis to new cancer was $40.2 \pm 25.8$ months in men and $42.3 \pm$ 27.2 months in women (Table 2). Liver cancer (20.6\%) was the most frequent cancer in men, followed by cancers of the bladder $(16.9 \%)$, kidney $(11.6 \%)$, colorectal $(10.8 \%)$, lung $(8.4 \%)$, and stomach $(7.4 \%)$ (Table 2). In women, bladder cancer $(25.6 \%)$ was the most frequent, followed by cancers of the kidney $(16.3 \%)$, breast $(11.2 \%)$, liver $(9.6 \%)$, colorectal $(9.7 \%)$, and lung (3.3\%).

We compared gender differences in incidence of site specific cancer. Women had a higher incidence rate of renal and urinary tract cancer. Men and women had a bladder cancer incidence rate of 224 and 322 per 100,000 person-years (PY), respectively. They had kidney cancer incidences of 154 and 206 per 100,000 PY, respectively. In contrast, the men had a higher incidence of liver, lung, and stomach cancers. Men and women had a liver cancer incidence rate of 274 and 121 per 100,000 PY, respectively. They had stomach cancer incidence rate of was 63 and 40 per 100,000 PY, respectively.

Table 1. Demographics of end-stage renal disease dialysis (ESRD) patients with and without new cancer, and results of a multivariate analysis of cancer risks.

\begin{tabular}{|c|c|c|c|}
\hline \multicolumn{4}{|c|}{$\begin{array}{l}\text { ESRD patients on maintenance dialysis, } N(\%)=40,833 \\
(100 \%)\end{array}$} \\
\hline & $\begin{array}{l}\text { Without new } \\
\text { cancer } 38481 \\
(94.2 \%)\end{array}$ & $\begin{array}{l}\text { With new cancer } \\
2352(5.8 \%)\end{array}$ & $\begin{array}{l}\text { Multivariate } \\
\text { Analysis } \\
\text { Adjusted HR } \\
(95 \% \text { CI })\end{array}$ \\
\hline \multicolumn{4}{|l|}{$\begin{array}{l}\text { Age at first dialysis } \\
\text { (years) }\end{array}$} \\
\hline$<35$ & $2280(97.6)$ & $55(2.4)$ & Reference \\
\hline $35-64$ & $21146(93.8)$ & $1397(6.2)$ & $3.42(2.60-4.48)^{* *}$ \\
\hline$\geq 65$ & $15055(94.4)$ & $900(5.6)$ & $4.73(3.59-6.25)^{* *}$ \\
\hline \multicolumn{2}{|c|}{$\begin{array}{l}\text { Age at cancer } \\
\text { diagnosed (years) }\end{array}$} & \multicolumn{2}{|l|}{$63.0 \pm 10.1^{*}$} \\
\hline \multicolumn{2}{|c|}{$\begin{array}{l}\text { Mean time from dialysis to cancer } \\
\text { (months) }\end{array}$} & \multicolumn{2}{|l|}{$41.3 \pm 26.6^{*}$} \\
\hline \multicolumn{2}{|c|}{$\begin{array}{l}\text { Median time from dialysis to cancer } \\
\text { (months) }\end{array}$} & \multicolumn{2}{|c|}{$\begin{array}{l}37.37 \text { (25th-75th percentile, } 19.37 \text { - } \\
58.19)\end{array}$} \\
\hline \multicolumn{4}{|c|}{ Gender } \\
\hline Female & $20211(94.2)$ & $1232(5.8)$ & Reference \\
\hline Male & $18270(94.3)$ & $1120(5.7)$ & $1.10(1.02-1.20)^{\star *}$ \\
\hline \multicolumn{4}{|l|}{$\begin{array}{l}\text { Initial dialysis } \\
\text { modality }\end{array}$} \\
\hline Hemodialysis & $34890(95.5)$ & $2181(4.5)$ & Reference \\
\hline Peritoneal dialysis & $3577(94.1)$ & $170(5.9)^{\#}$ & $0.88(0.75-1.21)$ \\
\hline \multicolumn{4}{|l|}{ Comorbidity } \\
\hline \multicolumn{4}{|l|}{ Diabetes mellitus } \\
\hline no & $19616(92.7)$ & $1534(7.3)^{\#}$ & Reference \\
\hline yes & $18865(95.8)$ & $818(4.2)$ & $0.74(0.67-0.81)^{\star *}$ \\
\hline \multicolumn{4}{|l|}{ Hypertension } \\
\hline no & $8173(91.7)$ & $735(8.3)^{\#}$ & Reference \\
\hline yes & $30310(94.9)$ & $1617(5.1)$ & $0.69(0.63-0.76)^{* *}$ \\
\hline \multicolumn{4}{|c|}{ Cardiovascular disease } \\
\hline no & $22810(93.3)$ & $1633(6.7)^{\#}$ & Reference \\
\hline yes & $15671(95.6)$ & $719(4.4)$ & $0.89(0.81-0.98)^{* *}$ \\
\hline \multicolumn{4}{|l|}{ Chronic lung disease } \\
\hline no & $34479(94.2)$ & $2138(5.8)$ & Reference \\
\hline yes & $4002(94.9)$ & $214(5.1)$ & $1.03(0.89-1.19)$ \\
\hline \multicolumn{4}{|l|}{ Chronic liver disease } \\
\hline no & 35474 (94.5) & $2070(5.5)^{\#}$ & Reference \\
\hline yes & 3007 (91.4) & $282(8.6)$ & $1.76(1.55-1.99)^{* *}$ \\
\hline \multicolumn{4}{|l|}{$\begin{array}{l}\text { Congenital renal and } \\
\text { urinary tract disease }\end{array}$} \\
\hline no & $37679(94.2)$ & $2320(5.8)$ & Reference \\
\hline yes & $802(96.2)$ & $32(3.8)$ & $0.77(0.68-1.23)$ \\
\hline
\end{tabular}




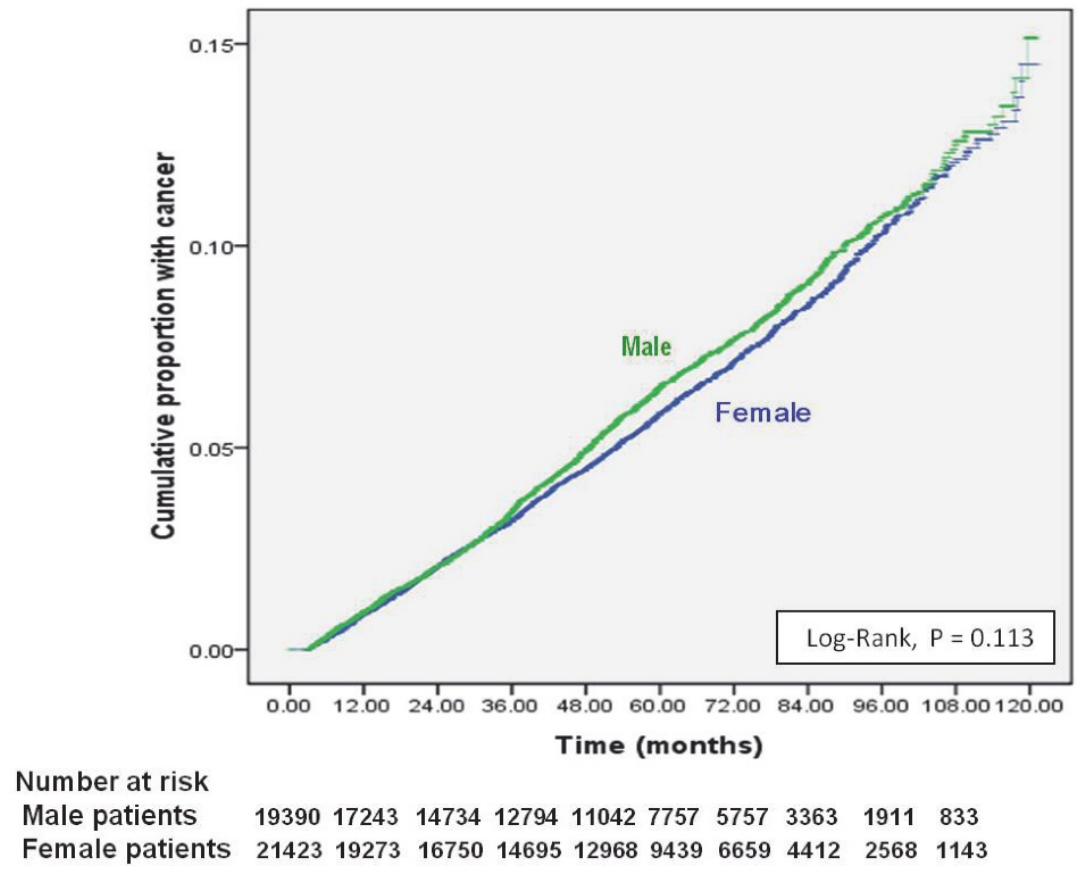

Figure 1. Cumulative incidence of new cancers from initial dialysis in end-stage renal disease dialysis patients, stratified by gender.

Table 2. Site-specific cancer incidence rate and 5-year survival rate of the most common cancers in end-stage renal disease dialysis patients (stratified by gender).

\begin{tabular}{|c|c|c|c|c|c|c|c|c|}
\hline & \multirow{2}{*}{$\frac{\text { Men }}{\mathrm{n}(\%)}$} & \multicolumn{7}{|c|}{ Women } \\
\hline & & $\begin{array}{l}\text { Mean time from dialysis } \\
\text { to cancer (months) }\end{array}$ & $\begin{array}{l}\text { Incidence } \\
\text { Rate }^{* *}\end{array}$ & $\begin{array}{l}5 \text {-year survival } \\
\text { rate }(\%)\end{array}$ & $\mathrm{n}(\%)$ & $\begin{array}{l}\text { Mean time from dialysis } \\
\text { to cancer (months) }\end{array}$ & $\begin{array}{l}\text { Incidence } \\
\text { Rate }^{* *}\end{array}$ & $\begin{array}{l}5 \text {-year survival } \\
\text { rate }(\%)\end{array}$ \\
\hline Total cancers & $1120(100 \%)$ & $40.2 \pm 25.8$ & 1328 & & $1232(100 \%)$ & $42.3 \pm 27.2$ & 1260 & \\
\hline Bladder & $189(16.9 \%)$ & $41.9 \pm 23.9$ & 224 & 52 & $315(25.6 \%)$ & $50.5 \pm 26.0$ & 322 & 59 \\
\hline Kidney & $130(11.6 \%)$ & $39.5 \pm 28.0$ & 154 & 69 & $201(16.3 \%)$ & $39.6 \pm 28.5$ & 206 & 73 \\
\hline Liver & $231(20.6 \%)$ & $39.6 \pm 25.1$ & 274 & 21 & $118(9.6 \%)$ & $37.4 \pm 25.4$ & 121 & 21 \\
\hline Colon & $121(10.8 \%)$ & $40.1 \pm 24.5$ & 144 & 32 & $119(9.7 \%)$ & $36.0 \pm 24.9$ & 122 & 47 \\
\hline Lung & $94(8.4 \%)$ & $42.5 \pm 24.9$ & 111 & 8 & $40(3.3 \%)$ & $47.3 \pm 29.6$ & 41 & 4 \\
\hline Stomach & $53(7.4 \%)$ & $38.7 \pm 27.6$ & 63 & 24 & $39(3.2 \%)$ & $23.8 \pm 28.0$ & 40 & 11 \\
\hline Mouth & $34(3.0 \%)$ & $33.5 \pm 23.0$ & 40 & & $9(0.7 \%)$ & $50.7 \pm 27.4$ & 9 & \\
\hline \multirow[t]{2}{*}{ Tongue } & $18(1.6 \%)$ & $40.2 \pm 25.3$ & 21 & & $7(0.6 \%)$ & $35.1 \pm 27.1$ & 7 & \\
\hline & $17(1.5 \%)$ & $46.6 \pm 31.5$ & 20 & & $5(0.4 \%)$ & $38.8 \pm 22.7$ & 5 & \\
\hline \multicolumn{9}{|c|}{ Nasopharynx } \\
\hline Thyroid & $12(1.1 \%)$ & $49.5 \pm 33.1$ & 14 & & $25(2.0 \%)$ & $49.0 \pm 29.8$ & 26 & \\
\hline Prostate & $44(3.9 \%)$ & $38.5 \pm 29.1$ & 52 & & - & - & & \\
\hline Breast & - & - & - & & $138(11.2 \%)$ & $40.6 \pm 27.6$ & 141 & 61 \\
\hline Cervix & - & - & - & & $63(5.1 \%)$ & $38.0 \pm 27.7$ & 64 & 24 \\
\hline
\end{tabular}

* Data are means \pm standard deviation (SD).

** 100,000 person-years.

We also estimated gender differences in 5-year survival rate of site specific cancer. The 5-yer survival rate for bladder cancer, kidney cancer, liver cancer, colon cancer, lung cancer, and stomach cancer was $52 \%, 69 \%, 21 \%, 32 \%, 8 \%$ and $24 \%$ in men and $59 \%$, $73 \%, 21 \%, 47 \%, 4 \%$ and $11 \%$ in women.

\section{Survival analysis and risk factors for mortality in dialysis patients with cancer}

Women had a better cumulative survival rate than men did in the cancer group. The 1-, 3-, 5-, and 7-year cumulative survival rates (Kaplan-Meier analysis) were $64.2 \%, 43.0 \%, 33.3 \%$, and $29.7 \%$ in men, and $74.4 \%, 57.6 \%, 49.8 \%$, and $44.7 \%$ in women (Figure 2 ). Women also had a better survival rate then male patients after bladder cancer had been diagnosed (log-rank: $\mathrm{P}=0.026$ ) (Figure 3a); however, there were no significant differences in survival between men and women after diagnoses of other cancers (Figure 3, 4). Women with kidney cancer had the best survival rates, followed by those with bladder, breast, cervix, colon, liver, stomach, and lung cancers. Men with kidney cancer also had the best survival rates, followed by bladder, colon, liver, stomach and lung cancers (Table 2).

Multivariate Cox proportional hazards analysis 
showed that being $\geq 35$, being male, having DM, and having CVD were independent predictors of all-cause mortality in patients with cancer (Table 3). Lung, nasopharyngeal, liver, stomach, tongue, mouth, cervical, colorectal, and bladder cancer had higher mortality rates than kidney cancer (reference group) (HR: 1). For example, the HRs for lung cancer were 6.31 (95\% CI: 4.70 to 8.48), nasopharyngeal cancer 5.81 (95\% CI: 3.42 to 9.85$)$, and liver cancer 3.92 (95\% CI: 3.02 to 5.09$)$.

Table 3. Risk factors for all-cause mortality in end-stage renal disease dialysis patients with cancer.

\begin{tabular}{|c|c|c|}
\hline Variables & Univariate Analysis & Multivariate Analysis \\
\hline & aHR $(95 \%$ CI) & aHR $(95 \% \mathrm{CI})$ \\
\hline \multicolumn{3}{|l|}{ Age at cancer diagnosis (years) } \\
\hline$<35$ & 1 (reference) & 1 (reference) \\
\hline $35-64$ & $2.60(1.34-5.02)^{*}$ & $2.25(1.16-4.38)^{*}$ \\
\hline$\geq 65$ & $5.00(2.59-9.66)^{*}$ & $3.66(1.88-7.12) * *$ \\
\hline Gender (Male vs. Female) & $1.53(1.36-1.72)^{*}$ & $1.16(1.02-1.32)^{*}$ \\
\hline Diabetes mellitus (yes vs. no) & $1.69(1.50-1.90)^{*}$ & $1.27(1.12-1.45) * *$ \\
\hline Hypertension (yes vs. no) & $1.30(1.14-1.48)^{*}$ & $0.89(0.77-1.02)$ \\
\hline Cardiovascular disease (yes vs. no) & $1.54(1.37-1.74)^{*}$ & $1.15(1.01-1.31)^{*}$ \\
\hline Chronic lung disease (yes vs. no) & $1.47(1.23-1.76)^{*}$ & $1.17(0.97-1.40)$ \\
\hline Chronic liver disease (yes vs. no) & $1.34(1.14-1.58)^{*}$ & $1.02(0.85-1.22)$ \\
\hline \multicolumn{3}{|l|}{ Cancer site } \\
\hline Kidney & 1 (reference) & 1 (reference) \\
\hline Bladder & $1.45(1.11-1.90)^{*}$ & $1.38(1.06-1.81)^{*}$ \\
\hline Liver & $4.37(3.39-5.62)^{*}$ & $3.92(3.02-5.09) * *$ \\
\hline Colon / Rectum & $2.68(2.02-3.55)^{*}$ & $2.15(1.61-2.86) * *$ \\
\hline Lung & $8.81(6.62-11.72)^{*}$ & $6.31(4.70-8.48) * *$ \\
\hline Stomach & $4.85(3.49-6.75)^{*}$ & $3.86(2.76-5.41) * *$ \\
\hline Mouth & $3.57(2.32-5.48)^{*}$ & $2.99(1.96-4.63) * *$ \\
\hline Tongue & $3.57(2.03-6.30)^{*}$ & $3.49(1.97-6.19)$ ** \\
\hline Nasopharynx & $5.64(3.34-9.52)^{*}$ & $5.81(3.42-9.85)$ ** \\
\hline Thyroid & $0.51(0.19-1.40)$ & $0.60(0.22-1.64)$ \\
\hline Prostate & $2.38(1.48-3.85)^{*}$ & $1.61(0.98-2.63)$ \\
\hline Breast & $1.30(0.90-1.88)$ & 1.35 (0.93-1.96) \\
\hline Cervix & $3.24(2.21-4.74)^{*}$ & $2.88(1.96-4.25)$ ** \\
\hline
\end{tabular}

$\mathrm{HR}$, hazard ratio; $\mathrm{CI}$, confidence interval.

aHR adjusted for gender, age, diabetes mellitus, hypertension, cardiovascular disease, chronic lung disease, and chronic liver disease.

$* \mathrm{P}<0.05$

** $\mathrm{P}<0.001$
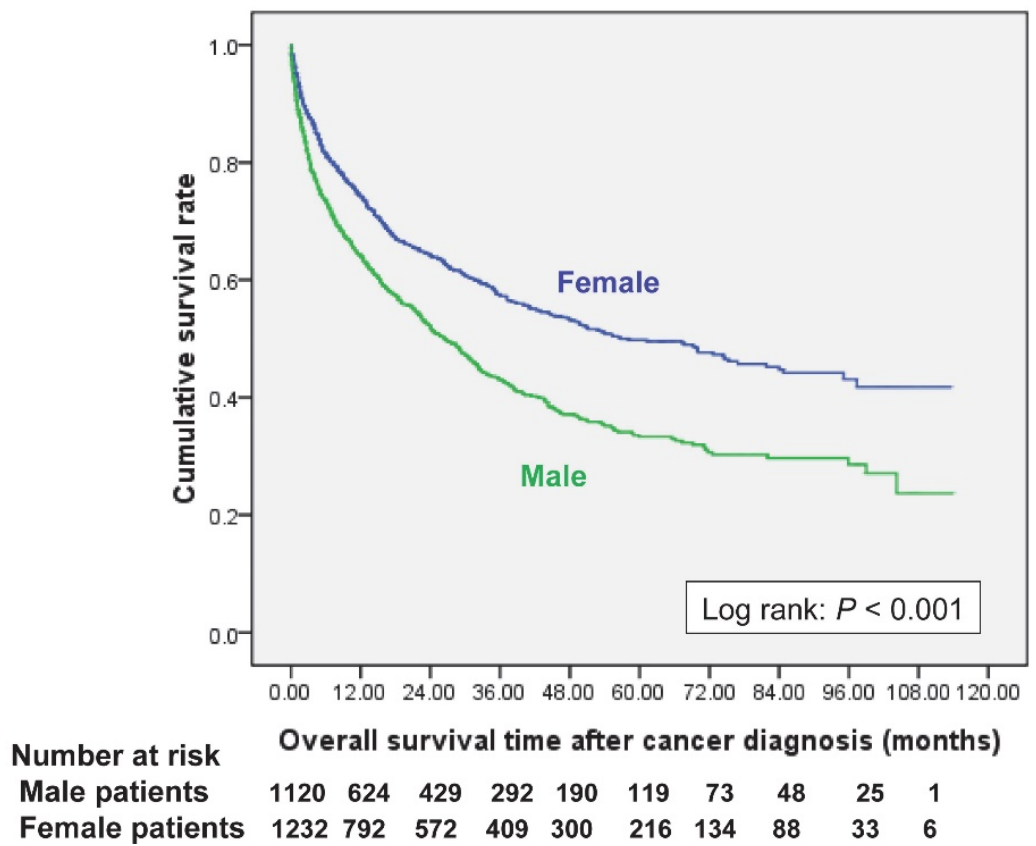

Figure 2. Overall survival curves after new cancer was diagnosed in end-stage renal disease dialysis patients, stratified by gender. 

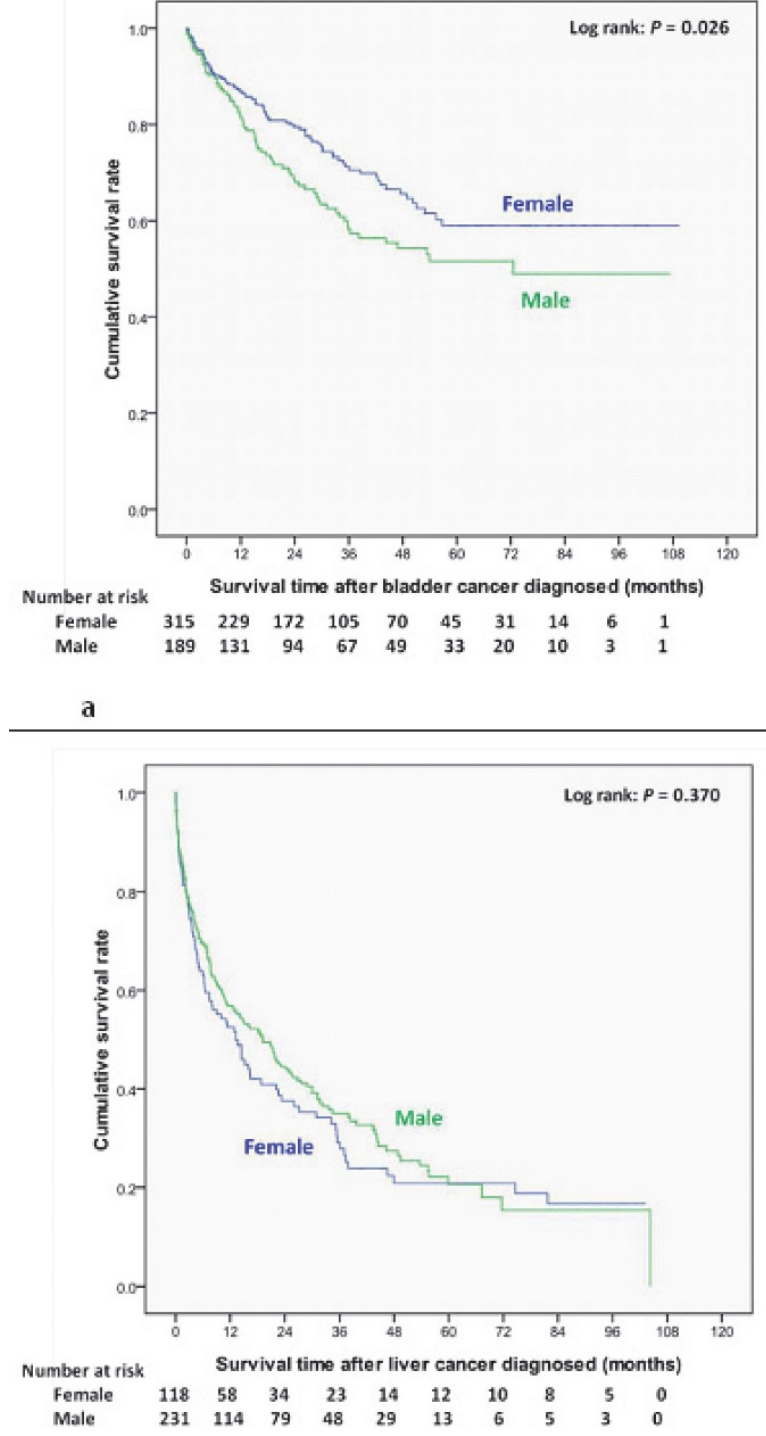

c.

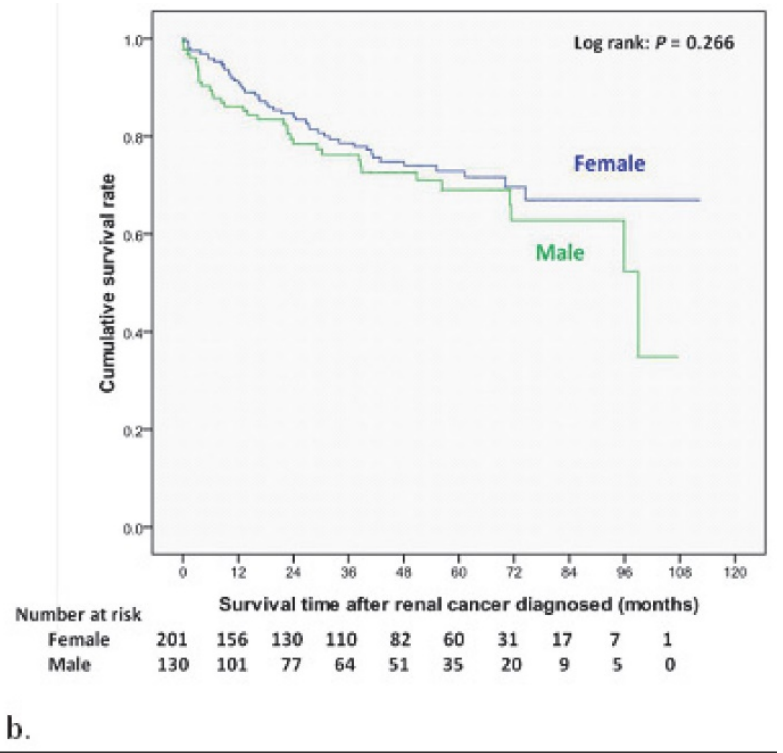

b.

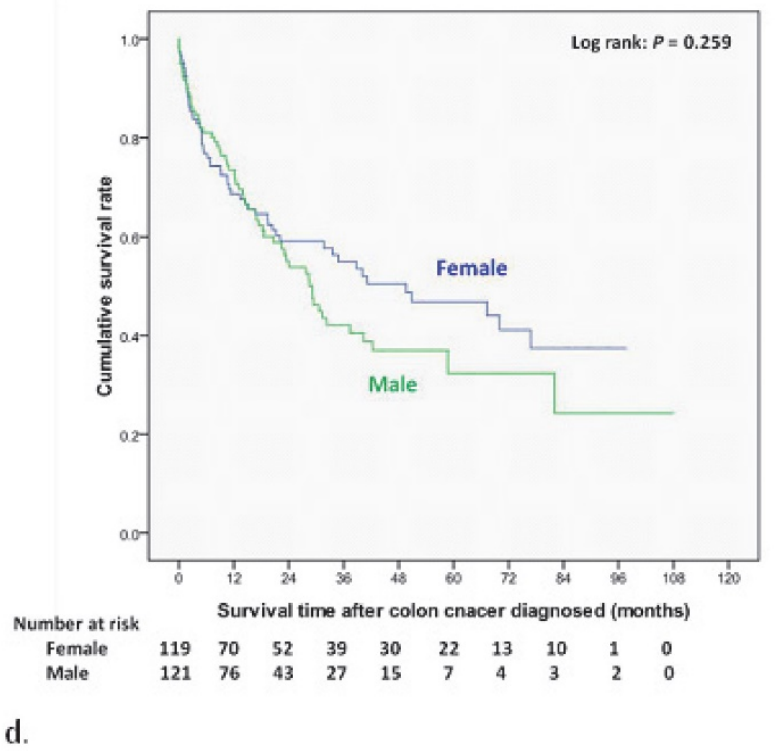

Figure 3. Overall survival curves of different site-specific cancers after newly diagnosed in end-stage renal disease dialysis patients. (a) Bladder cancer. (b) Kidney cancer. (c) Liver cancer. (d) Colon cancer.

\section{Discussion}

This study used the Taiwan's NHIRD to investigate the epidemiology and mortality of cancer in ESRD patients on dialysis. There were significant differences between men and women in the spectrum of cancer specific-sites. Bladder, kidney, and breast cancers were the three most frequent cancers in female ESRD dialysis patients; while, liver, bladder, and kidney cancers were the three most frequent cancers in male ESRD dialysis patients. We also analyzed the gender differences in 5-year survival rate of site specific cancer. The 5-yer survival rates of bladder cancer, kidney cancer, liver cancer, colon cancer, lung cancer, and stomach cancer were $52 \%$, $69 \%, 21 \%, 32 \%, 8 \%$ and $24 \%$ in men and $59 \%, 73 \%$,
$21 \%, 47 \%, 4 \%$ and $11 \%$ in women, respectively.

Compared with the general population, ESRD patients in Taiwan are a greater risk for various cancers $[5,17]$. Using randomly frequency-matching with an ESRD cohort and the general population, Liang et al. [5] found ESRD patients to be at a higher risk of developing overall cancer (HR: 1.64, 95\% CI: 1.54-1.74). Lin et al. [17] further reported patients receiving long-term dialysis were significant risk for all cancers (HR: 3.43, 95\% CI: 3.02-3.88) after consideration for the competing risk of mortality. They [17] also reported significant higher site-specific cancer risks in dialysis population, particularly in the development of oral, colorectal, liver, blood, breast, renal, bladder cancer.

The incidence of different cancers varies by 
geographic area worldwide [18]. The 2008 annual report of the Taiwan National Cancer Institute [19] reports liver, lung, and colorectal cancers to be the three most frequent in males in the general population; however, we found that liver, bladder, and kidney cancers to be the three most frequent in male ESRD patients on dialysis. Breast, colorectal, and lung cancers were the most frequent in females in the general population in Taiwan [14]; however, we found bladder, kidney, and breast cancers were the three most frequent in female ESRD patients on dialysis. Aristolochic acid (AA) is a major nephrotoxin, which induces interstitial nephritis and often ESRD [20-22]. AA is also a strong carcinogen for the renal pelvis, ureter, and bladder [23]. There is evidence of a causal relationship between AA exposure and nephropathy as well as urotract cancer. Histologic studies of AA nephropathy found interstitial fibrosis with atrophy and malignant uroepithelial cells [22]. Lai et al. found a dose-dependent relationship between consumption of AA-containing Chinese herbal products and an increased risk of cancer of the urinary tract in the general population in Taiwan [24]. Wang et al. also found an association between consumption of AA-related Chinese herbal products and an increased risk of developing urothelial cell carcinoma in Taiwan ESRD patients, especially women age 50 years and younger $[25,26]$.

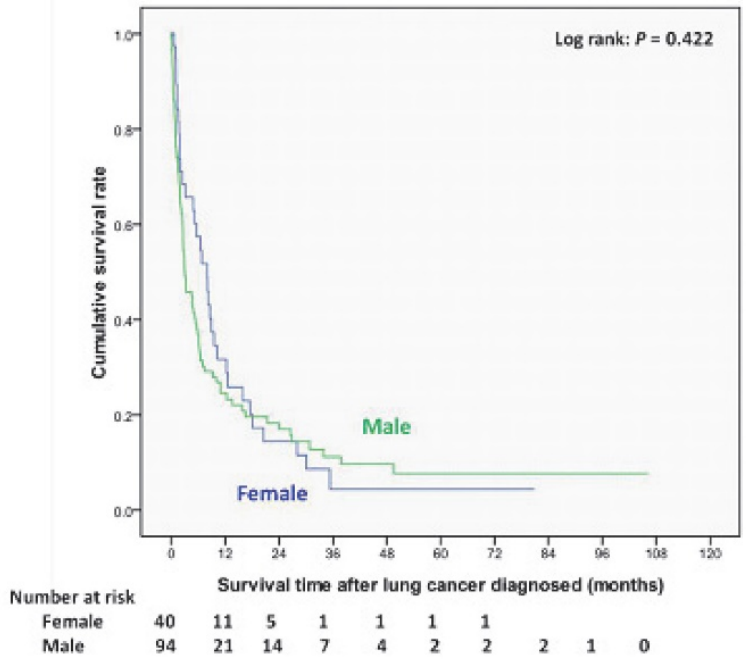

a.

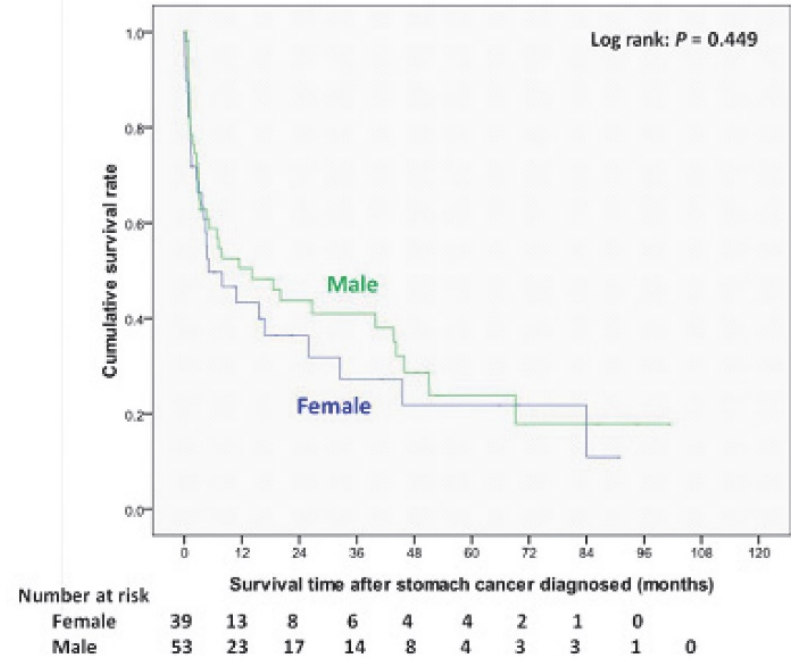

b.
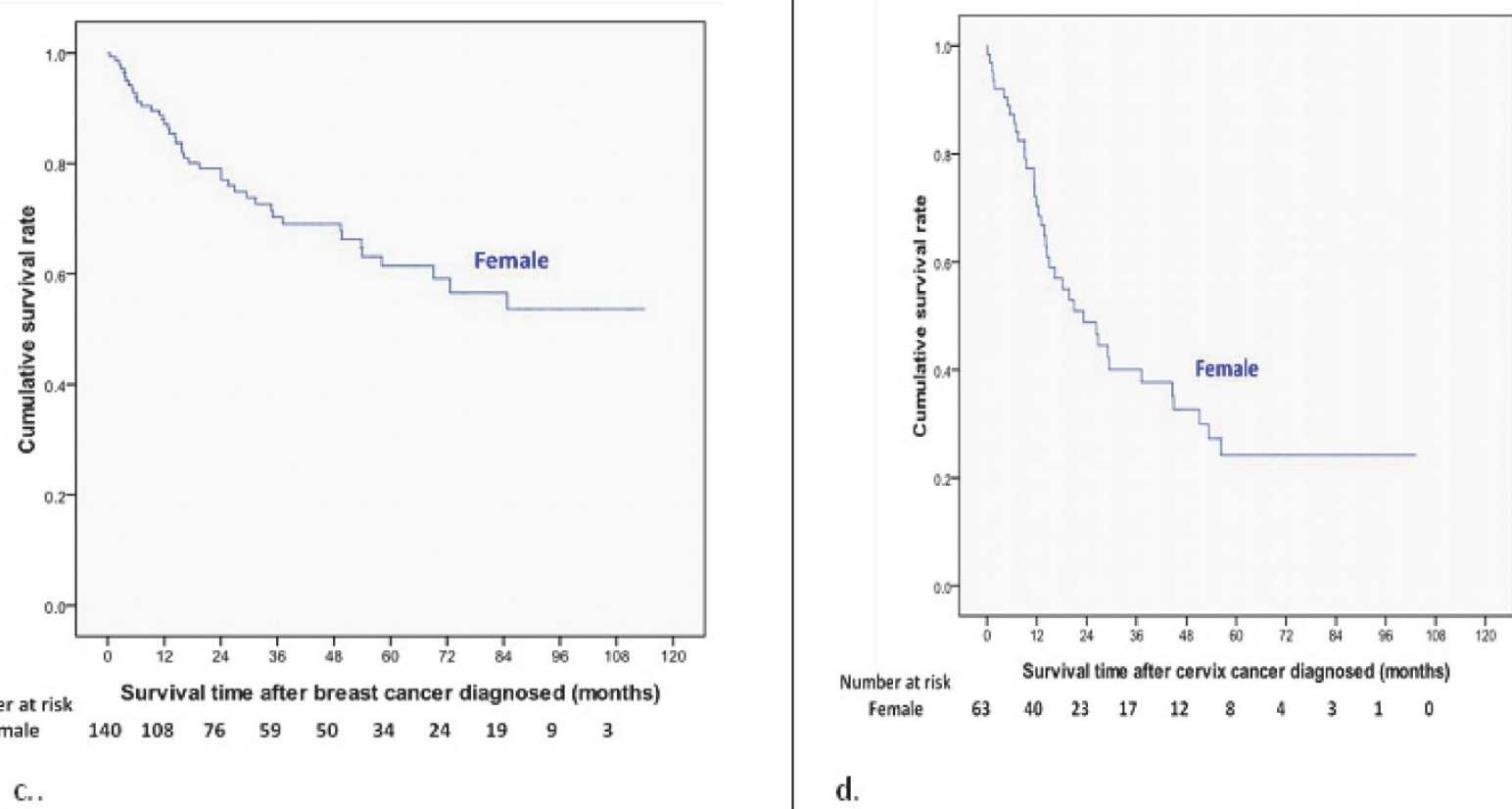

d.

Figure 4. Overall survival curves of different site-specific cancers after newly diagnosed in end-stage renal disease dialysis patients (a) Lung cancer. (b) Stomach cancer. (c) Breast cancer. (d) Cervix cancer. 

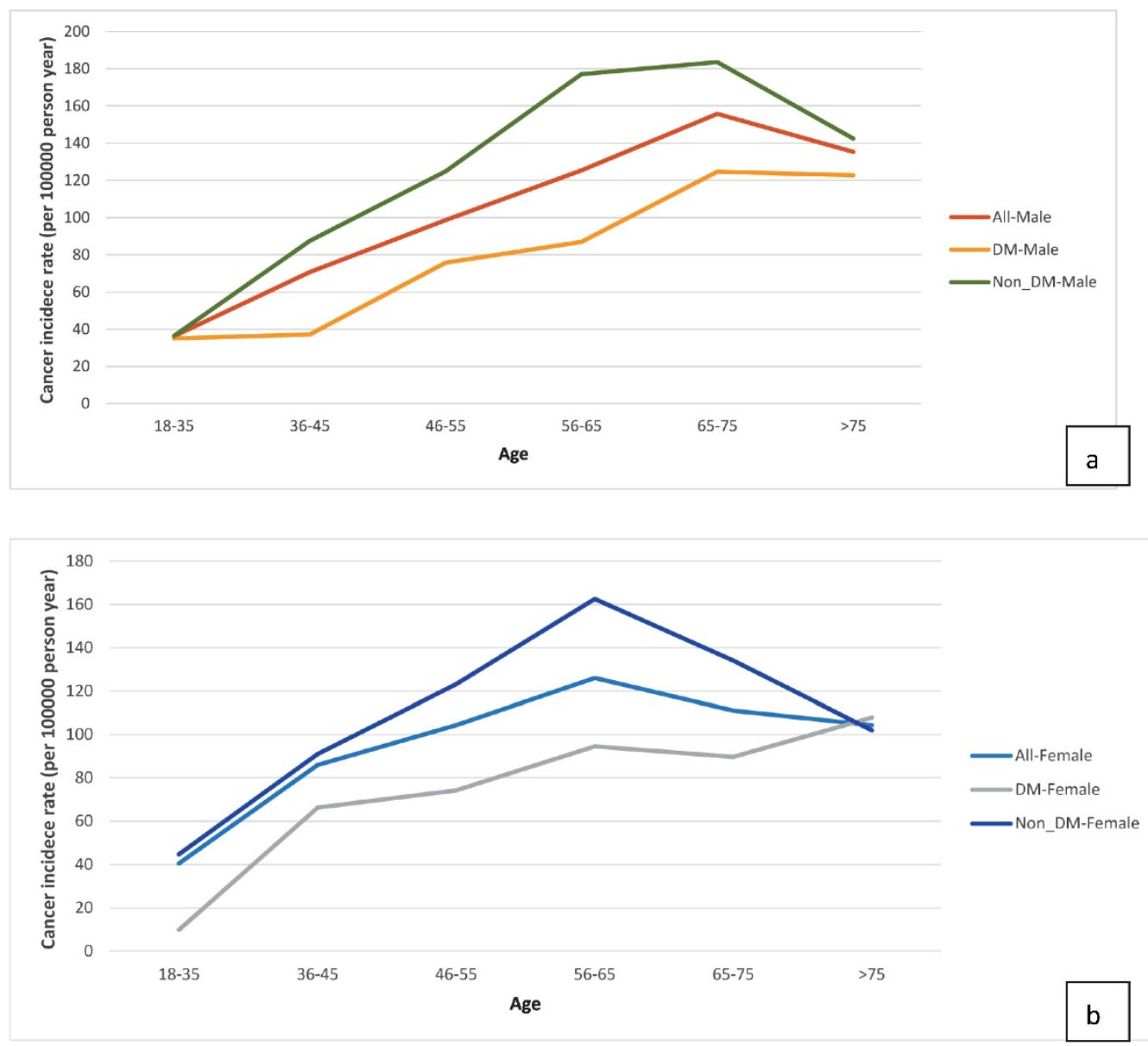

Figure 5. Age-specific cancer incidence stratified by diabetes mellitus status (a) Male ESRD patients. (b) Female ESRD patients.

We sought to identify risk factors for cancer in ESRD patients on dialysis and found that being older ( $\geq 65$ years) was one such risk factor, a finding consistent with other studies $[2,6,7]$. DM is a risk factor for cancer in the general population; whether $\mathrm{DM}$ is associated with a higher incidence of cancer in ESRD patients on dialysis is still being debated. Vajdic et al. [4] reported that patients with comorbid DM and on dialysis did not have a higher incidence of all-cancer; however, their study excluded patients with myeloma, kidney, and urinary tract cancers, which are known to frequently cause ESRD. In an international collaborative study [3], DM was found to be a significant risk factor for kidney cancer in Europe and the US. Our analysis showed that ESRD patients with DM had a lower risk for cancer after beginning dialysis. Possible causes for the inverse association are survival bias and time discrepancies between competing risk factors [27]. Because ESRD patients on dialysis have specific survival processes with short life span, their characteristics are different from those of the general population. Many ESRD patients with comorbid DM will not live long enough to develop cancers after beginning dialysis. One explanation for this is that ESRD patients have a high mortality rate because many of them have severe and complex comorbidities, such as DM, HTN, and CVD [27]. We further examined the incidence of age-specific cancers (Fig 5a \& 5b). In the real-world data analysis, the incidence of cancer was higher in ESRD patients without DM than those with DM across all the age-specific groups between 18 and 75 years. It would be better to compare them with a corresponding rate in the general Taiwanese population; however, the database of our study only included ESRD dialysis population. Further studies needed to be evaluated this issue.

Chronic liver disease is a common and serious worldwide medical problem. Compared with the general population, ESRD patients have a higher risk of a hepatitis virus infection $[9,28]$. For a variety of reasons, ESRD patients have risks of hepatitis $B$ virus (HBV) and hepatitis $C$ virus (HCV) acquisition $[9,28]$. First, patients with CKD have impaired host defenses against viral infection. Second, ESRD dialysis patients have a higher risk to contact this infection. HBV and $\mathrm{HCV}$ transmission among patients undergoing HD session has been reported. The time from initiation of 
dialysis (dialytic age) has been demonstrated as an independent risk factor. Several outbreaks of virus hepatitis in HD units were associated with lapses in infection control practice of renal care provider $[9,28]$. Chronic hepatitis is an independent risk factor for death primarily from liver cancer [29]. The risk for developing liver cancer is significantly higher in ESRD patients than in the general population [5]. We found a $76 \%$ higher risk of cancer in dialysis patients with chronic liver disease compared to those without (HR 1.76, 95\% CI: 1.55-1.99). Liver cancer was the most frequent cancer in men and the third most frequent in women on long-term dialysis, which may be related to the fact that - hepatitis $B$ and $C$ are endemic in Taiwan $[9,10,30]$ and because there is a higher risk of hepatitis viral infection among ESRD dialysis patients. Therefore, strategies to prevent HBV and $\mathrm{HCV}$ transmission are important for renal care provider in HD unit. Adherence to in infection control practice, such as staff education, environmental cleaning and disinfection, and proper medication handling. Vaccination and screening of patients and renal care provider in HD unit are also needed. For the patients who had been infected, treatment of virus hepatitis maybe considered.

There are few survival analyses and studies of the all-cause mortality of cancer in ESRD patients on dialysis [6]. In our site-specific cancer analyses, the 5 -yer survival rate of bladder cancer, liver cancer, colon cancer, lung cancer, and stomach cancer was $52 \%, 21 \%, 32 \%, 8 \%$ and $24 \%$ in men and $59 \%, 21 \%$, $47 \%, 4 \%$ and $11 \%$ in women, respectively. The survival rates of site-specific cancers in ESRD dialysis patients were much lower than those in general population. The 5-year survival rates of bladder cancer, liver cancer, colon cancer, lung cancer, and stomach cancer are $59.4 \%, 28 \%, 62.3 \%, 16.3 \%, 29.7 \%$ in general population [31]. The reasons for the different survival rates between ESRD and general population may be related to differences in the distribution of different cancer stages or the inability of ESRD patients to tolerate treatment due to aging and multi-comorbidities. One limitation of our study was that the NHIRD lacks cancer stage. Thus, more studies are needed to explore these differences.

The incidence of nasopharyngeal cancer is higher among ethnic Chinese than in the general populations of the US and Europe [32]. Nasopharyngeal cancer has a high mortality rate and a poor prognosis $[18,32], 5.81$ times higher than other cancers. Liver cancer is the third leading cause of cancer-related death in the general population [18]. Most patients with liver cancer also have coexisting liver cirrhosis [33]. Although considerable progress has been made in treating liver cancer in the past decades, liver cancer has a high mortality rate [33]. One recent study [34] showed higher mortality caused by liver cancer in CKD patients to not be dependent upon dialysis. We also found a high mortality rate for patients with ESRD on dialysis and comorbid liver cancer (5-year cumulative survival rate: $21 \%$ ).

\section{Clinical implications}

The problem of cancer in ESRD patients on dialysis is usually ignored because of the overwhelming number of mortalities due to cardiovascular and infectious diseases in this population. However, cancer is becoming much more important with the progressive aging of dialysis patients and their prolonged life spans, especially for high-risk patients and high-frequency cancers. Our results confirm what is known from other studies in North America and Europe, but they also highlight some unique features of the Taiwanese population on dialysis, namely high incidence of liver, colon, breast, and nasopharyngeal cancers. The distribution of site-specific cancers is different between men and women in patients on dialysis. In addition, more attention should be paid to teaching dialysis patients how to avoid the well-known cancer risks and carcinogens, including chronic hepatic virus infection, AA-containing herbs, etc. Finally, regular cancer screenings may need to be individualized, especially for high-risk patients.

\section{Limitations}

Our study has several limitations. One limitation is that the database of our study only included ESRD dialysis population, not the general Taiwanese population. It would be better to compare our patients with those in the general Taiwanese population. Another limitation was that our study lacked specific data on smoking status and cancer stage distribution.

\section{Conclusion}

The distribution of site-specific cancer was different between men and women in patients with ESRD on dialysis. More attention should be paid to teaching dialysis patients how to avoid the well-known cancer risks and carcinogens and individualized regular cancer screenings.

\section{Authors' contributions}

SFW, HYK, and CCC (Chien) designed the study. CCC (Chien), MMH, CCR, JJW, CCC (Chu), CYH, YMS, NCY, CHH, CCL and SFW collected data, analyzed the data and drafted the manuscript. CCC (Chien), YHC, MMH, CYH, YMS, NCY, CCLand SFW critically revised the manuscript for important intellectual content. 


\section{Acknowledgment}

This study was supported by grant CMFHR10402 from Chi-Mei Medical Center, grant NHRI-NHIRD-99182 and NHRI-NHIRD-101111from the Taiwan National Health Research Institutes, and grant KMU-Q105015 from Kaohsiung Medical University Research Foundation.

\section{Competing interests} interests.

The authors declare that they have no competing

\section{References}

1. Miao EY. Basilar invagination managed with electroacupuncture and Chinese herbal medicine: the report of a rare case in a pediatric patient. J. Altern. Complement. Med. 2009; 15: 197-200.

2. Wong G, Hayen A, Chapman JR, et al. Association of CKD and cancer risk in older people. J Am Soc Nephrol.2009; 20: 1341-50.

3. Maisonneuve P, Agodoa L, Gellert R, et al. Cancer in patients on dialysis for end-stage renal disease: an international collaborative study. Lancet. 1999; 354: 93-9.

4. Vajdic CM, McDonald SP, McCredie MR, et al. Cancer incidence before and after kidney transplantation. JAMA. 2006; 296:2823-31.

5. Liang JA, Sun LM, Yeh JJ, et al. The association between malignancy and end-stage renal disease in Taiwan. Jpn J Clin Oncol. 2011; 41: 752-7.

6. Lee JE, Han SH, Cho BC, et al. Cancer in patients on chronic dialysis in Korea. J Korean Med Sci. 2009; 24 Suppl:S95-S101.

7. Taneja S, Mandayam S, Kayani ZZ, Kuo YF, et al. Comparison of stage at diagnosis of cancer in patients who are on dialysis versus the general population. Clin J Am Soc Nephrol. 2007; 2: 1008-13.

8. Yang WC, Hwang SJ, Taiwan Society of Nephrology. Incidence, prevalence and mortality trends of dialysis end-stage renal disease in Taiwan from 1990 to 2001: the impact of national health insurance. Nephrol. Dial. Transplant. 2008; 23: 3977-82.

9. Patel PR, Thompson ND, Kallen AJ, et al. Epidemiology, surveillance, and prevention of hepatitis $\mathrm{C}$ virus infections in hemodialysis patients. Am J Kidney Dis. 2010; 56: 371-8.

10. Merican I, Guan R, Amarapuka D, et al. Chronic hepatitis B virus infection in Asian countries. J Gastroenterol Hepatol. 2000; 15:1356-61.

11. Chien CC, Yen CS, Wang JJ, et al. Reverse epidemiology of hypertension-mortality associations in hemodialysis patients: a long-term population-based study. Am J Hypertens. 2012; 25:900-6.

12. Tien $\mathrm{KJ}$, Lin ZZ, Chio CC, et al. Epidemiology and mortality of new-onset diabetes after dialysis: Taiwan national cohort study. Diabetes Care. 2013; 36: 3027-32.

13. Hong CS, Chung KM, Huang PC, et al. Epidemiology and mortality of liver abscess in end-stage renal disease dialysis patients: Taiwan national cohort study. PLoS One. 2014; 9: e88078.

14. Lin ZZ, Wang JJ, Chung CR, et al. Epidemiology and mortality of hip fracture among patients on dialysis: Taiwan National Cohort Study. Bone. 2014; 64: 235-9.

15. Chou MT, Wang JJ, Wu WS, et al. Epidemiologic features and long-term outcome of dialysis patients with infective endocarditis in Taiwan. Int J Cardiol. 2015; 179:465-9.

16. Kan WC, Wang JJ, Wang SY, et al. The new comorbidity index for predicting survival in elderly dialysis patients: a long-term population-based study. PLoS One. 2013; 8:e68748.

17. Lin MY, Kuo MC, Hung CC, et al. Association of dialysis with the risks of cancers. PLoS One. 2015; 10:e0122856.

18. Parkin DM, Bray F, Ferlay J, et al. Estimating the world cancer burden: Globocan 2000. Int J Cancer. 2001; 94: 153-6.

19. Wang X, Liang XB, Li FQ, et al. Therapeutic strategies for Parkinson's disease: the ancient meets the future--traditional Chinese herbal medicine, electroacupuncture, gene therapy and stem cells. Neurochem. Res. 2008; 33: 1956-63.

20. Vanherweghem JL, Depierreux M, Tielemans C, et al. Rapidly progressive interstitial renal fibrosis in young women: association with slimming regimen including Chinese herbs. Lancet. 1993; 341:387.

21. Depierreux M, Van Damme B, Vanden Houte K, et al. Pathologic aspects of a newly described nephropathy related to the prolonged use of Chinese herbs. Am J Kidney Dis. 1994; 24:172.

22. Debelle FD, Vanherweghem JL, Nortier JL. Aristolochic acid nephropathy: a worldwide problem. Kidney Int. 2008; 74:158.

23. Yang HY, Chen PC, Wang JD. Chinese herbs containing aristolochic acid associated with renal failure and urothelial carcinoma: a review from epidemiologic observations to causal inference. Biomed Res Int. 2014; 569325.
24. Lai MN, Wang SM, Chen PC, et al. Population-based case-control study of Chinese herbal products containing aristolochic acid and urinary tract cancer risk. J Natl Cancer Inst. 2010; 102:179-86.

25. Wang SM, Lai MN, Wei A, et al. Increased risk of urinary tract cancer in ESRD patients associated with usage of Chinese herbal products suspected of containing aristolochic acid. PLoS One. 2014; 9: e105218.

26. Wang SM, Lai MN, Chen PC, et al. Increased risk of urothelial cancer in young and middle aged patients with end-stage renal disease. J Formos Med Assoc. 2015; 114: 52-7.

27. Kalantar-Zadeh K, Block G., Humphreys M H, et al. Reverse epidemiology of cardiovascular risk factors in maintenance dialysis patients. Kidney Int. 2003; 63: 793-808.

28. Edey M, Barraclough K, Johnson DW. Review article: Hepatitis B and dialysis. Nephrology. 2000; 15:137-45.

29. Perico N, Cattaneo D, Bikbov B, et al. Hepatitis C infection and chronic renal diseases. Clin J Am Soc Nephrol. 2009; 4: 207-20.

30. Chen CI, You SL, Lin LH, et al. Cancer epidemiology and control in Taiwan: a brief review. Jpn J Clin Oncol. 2002; 32( Suppl): S66-S81.

31. Taiwan Cancer Registry. http://tcr.cph.ntu.edu.tw/main.php?Page=N1.

32. Jeyakumar A, Brickman TM, Jeyakumar A, et al. Review of nasopharyngeal carcinoma. Ear Nose Throat J. 2006; 85:168-70.

33. Rampone B, Schiavone B, Martino A, et al. Current management strategy of hepatocellular carcinoma. World J Gastroenterol. 2009; 15: 3210-16.

34. Weng PH, Hung KY, Huang HL, et al. Cancer-specific mortality in chronic kidney disease: longitudinal follow-up of a large cohort. Clin J Am Soc Nephrol. 2001; 6: 1121-28. 